\title{
Single-chain nanoparticles $v s$. star, hyperbranched and dendrimeric polymers: Effect of the nanoscopic architecture on the flow properties of diluted solutions
}

\author{
Irma Perez-Baena, ${ }^{a b}$ Angel J. Moreno ${ }^{a b}$, Juan Colmenero ${ }^{a b c d}$ and José A. \\ Pomposo*abce
}

\begin{abstract}
The flow properties of dilute solutions of linear, star, hyperbranched and dendrimeric polymers have been the subject of numerous studies. However, no systematic analysis has been carried out for the case of single-chain nanoparticles (SCNPs) of different nature, which are unimolecular soft nanoobjects consisting of individual polymer chains collapsed to a certain degree by means of intramolecular bonding. On the basis of the fractal nature of SCNPS and experimental data of the hydrodynamic radius, a simple predictive power-law between the intrinsic viscosity and molecular weight is proposed. Furthermore, a comparison is made between the intrinsic viscosities of the SCNPS and of low-functionality stars, hyperbranched and dendrimeric polymers of the same chemical nature and molecular weight. As a consequence of their complex nanoscopic architecture, the intrinsic viscosities of SCNPs are systematically smaller than those of linear chains and low-functionality stars. When compared with hyperbranched and dendrimeric polymers, a complex behaviour is found, this being highly dependent on the molecular weight and amount of X-linkers of the SCNP.
\end{abstract}

\section{Introduction}

A key parameter providing the frictional contributions of polymers of different architecture (linear, star, hyperbranched, etc.) in diluted solutions is the intrinsic viscosity, $[\eta]$, which measures the polymer's ability to increase the viscosity of a solvent. ${ }^{1,2}$ The intrinsic viscosity is defined as the ratio of the increase in the relative viscosity $\left(\eta / \eta_{\mathrm{s}}\right)$ by the polymeric solute to its concentration $c$ in the limit of infinite dilution: $[\eta]=$ $\lim _{c \rightarrow 0}\left(\eta-\eta_{s}\right) / c \eta_{s}$. In the former expressions $\eta$ and $\eta_{\mathrm{s}}$ are the viscosities of the solution and the pure solvent, respectively.

Even if a huge amount of work has been devoted to investigate the flow properties in dilute solution of polymers with complex topologies like star, hyperbranched, and dendrimeric polymers, no systematic analysis has been carried out yet for the case of single-chain polymer nanoparticles (SCNPs), which are individual polymer chains collapsed to a certain degree by means of intramolecular bonding. SCNPs are unimolecular soft nano-objects in the sub-20 $\mathrm{nm}$ range with potential applications in catalysis, sensing and drug delivery. ${ }^{3-6}$ Very recently, SANS and SAXS measurements, ${ }^{7-9}$ as well as complementary coarse-grained $\mathrm{MD}$ simulations, ${ }^{10,11}$ have revealed that SCNPs in solution adopt open, sparse morphologies resembling those of intrinsically disordered proteins with locally compact portions connected by flexible segments. The effect of the complex, non-controllable topology of SCNPs on the behaviour of their intrinsic viscosity seems a highly non-trivial problem, given the deep impact already observed in systems of controllable architecture as, e.g., linear, star, or dendrimeric polymers.
In the case of linear polymers, the $\mathrm{Zimm}_{\text {model }}{ }^{12}$ provides a convenient support to the well-known Fox-Flory equation: $[\eta]$ $=\Phi\left(6^{1 / 2} R_{g}\right)^{3} / M$, which relates $[\eta]$ with the radius of gyration, $R_{g}$, and the molecular weight, $M$. The factor $\Phi$ is a universal constant. $^{1,2}$ By assuming a power-law dependence of the polymer size on the number of monomers, $R_{g} \propto M^{v}$ (i.e., selfsimilarity, fractal behavior), the Fox-Flory equation leads to the Mark-Houking-Sakurada (MHS) equation: $[\eta]=K_{\eta} M^{a}$, where $K_{\eta}$ and $a=3 v-1$ are constants for a given polymer-solvent pair. ${ }^{2}$ The exponent $a$ in the MHS equation is a constant whose value depends on the macromolecular architecture and the solvent quality. Linear polymers in $\Theta$-solvent adopt gaussian conformations ( $v=0.5$ ), and therefore $a=0.5$. Linear polymers in ideal good solvent conditions are self-avoiding random walks scaling with the Flory exponent $v_{\mathrm{F}} \approx 0.59 .^{1,2}$ Accordingly, the expected scaling exponent for $[\eta]$ in good solvent is $a \approx 0.76$.

Star polymers with different arm numbers also follow the MHS equation. For this particular polymer architecture, the value of the exponent in the MHS equation is very similar to that displayed by linear chains of the same chemical nature, ${ }^{12-14}$ and must be identical in the limit of large molecular weight. ${ }^{2}$ However, for a fixed value of $M$, the value of $[\eta]$ decreases upon increasing the arm number $f$, because of the inverse dependence of the star size on $f$. This behavior has been observed for star polymers both in $\theta$-solvent ${ }^{15,16}$ and good solvent conditions. ${ }^{15,17}$

Hyperbranched polymers with long spacer length also follow the MHS equation ${ }^{18}$ indicating that these chains with such particular topology are fractal objects. ${ }^{2,18}$ For these systems, the exponent in the MHS equation takes values below 0.5 (e.g., 0.39 for hyperbranched PS chains with long spacers) 
and vary with the molecular weight of the spacer. ${ }^{18}$ For fixed $M,[\eta]$ increases on increasing the spacer length. For comparison, hyperbranched polymers with short spacer length show values of the exponent $a$ in the range of $0.3-0.5{ }^{19}$

Dendrimeric polymers, on the contrary, are not self-similar and they do not follow the MHS equation. ${ }^{20,21}$ In fact, dendrimeric polymers usually show a maximum (i.e., bellshaped curve) in the classical $[\eta]$ vs. generation number $(G)$ plot. This has been explained on the basis of the Einstein's result $^{22}$ for hard spheres, $[\eta] \propto V_{H} / M$, and simple scaling arguments for $M$ and the hydrodynamic volume $V_{H}$. Namely, the molecular weight in dendrimeric polymers with a branch multiplicity $B$ increases exponentially with $G$ according to $M \propto$ $B^{G}$, whereas the hydrodynamic volume grows with $G$ as $V_{H} \propto$ $G^{3}$. Therefore, the intrinsic viscosity scales as $[\eta] \propto G^{3} / B^{G}$, which is a nonmonotonic function in the generation number. Thus, $[\eta]$ for dendrimeric polymers shows a maximum at $G=$ $3 / \ln (B) .^{23,24}$

SCNPs in solution, according to recent SANS and SAXS experiments as well as complementary MD simulations, behave as fractal objects ${ }^{25}$ following a power-law relation $R_{H}=K_{H} M^{v}$, where $R_{H}$ is the hydrodynamic radius and $K_{H}$ is a systemdependent constant related to the statistical segment size. ${ }^{1,2}$ The particular value of the scaling exponent $v$ shows some dependence on the amount of reactive cross-linker (X-linker) functional groups in the precursor. ${ }^{25}$ For instance, poly(methyl methacrylate) (PMMA)- and poly(styrene) (PS)-SCNPs synthesized from precursors with $20 \mathrm{~mol} \%$ of X-linker functional groups, showed $v$-values of 0.48 and 0.50 , respectively, when using $R_{H}$ data from size exclusion chromatography (SEC) measurements. On the other hand, PSSCNPs synthesized from precursors with 5, 15 and $30 \mathrm{~mol} \%$ of $\mathrm{X}$-linker functional groups displayed $v$-values of $0.52,0.49$ and 0.47 , respectively.

Based on these results, in this work we derive simple scaling power-laws between $[\eta]$ and $M$ as a function of the amount of X-linkers. Next, we perform a comparison of the values of $[\eta]$ derived from these expressions to experimental data available for a variety of SCNPs of different chemical nature. Finally, a quantitative comparison is performed of $[\eta]$ values for SCNPs and for low-functionality star, hyperbranched and dendrimeric polymers, of the same chemical nature and molecular weight, in order to unravel the effect of the nanoscopic architecture on the flow properties of diluted solutions of polymers with different architectures. We find that, as a consequence of their complex nanoscopic architecture, the intrinsic viscosities of SCNPs are systematically smaller than those of linear chains and low-functionality stars. When compared with hyperbranched and dendrimeric polymers, a complex behaviour is found, this being highly dependent on the molecular weight and the amount of X-linkers of the SCNP.

\section{SCNPs: effect of the nanoscopic architecture on the intrinsic viscosity}

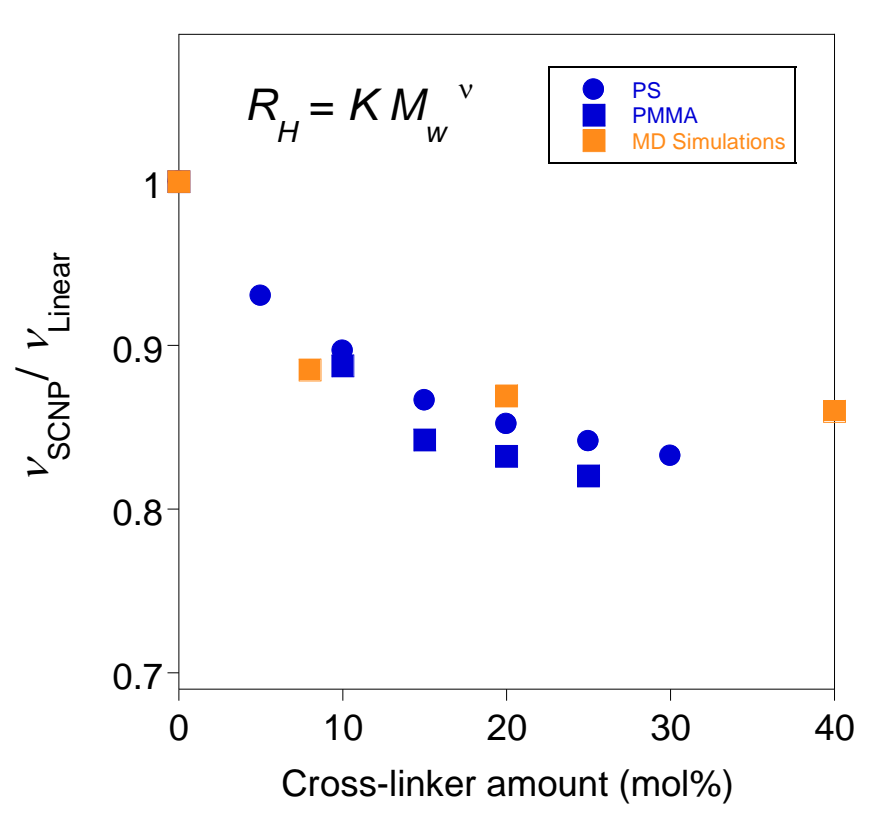

Fig. 1. Evolution of the ratio of the exponent $v$, in the power-law $R_{H}=K_{H} M^{v}$ of SCNPS, to the corresponding value of the precursor, as a function of the X-linker amount in the precursor for: PS-SCNPs (blue circles), and PMMA-SCNPs (blue squares). $R_{H}$ data were obtained from SEC measurements (see ref. 25). Data from $\mathrm{MD}$ simulations of a generic bead-spring model for SCNPs ${ }^{10}$ are extracted from $<R_{g}^{2}>^{1 / 2} \propto M^{v}$ fits, and are also shown for comparison (orange squares).

As revealed by MD simulations ${ }^{10,11}$ and consistently with scattering experiments, ${ }^{7-11,25}$ SCNPs in solution adopt open, sparse morphologies resembling those of intrinsically disordered proteins with locally compact portions connected by flexible segments. This is confirmed by compiling literature data for hydrodynamic radii of SCNPs, ${ }^{25}$ which are consistent with scaling exponents $v \sim 1 / 2$, similar to those of chains in $\Theta-$ solvent or intrinsically disordered proteins, and rather different from those of globular proteins $(v \sim 1 / 3)$. The precise value of the exponent $v$ shows some dependence on the amount of $\mathrm{X}$ linkers. Figure 1 shows the ratio of the value of the exponent $v$ for PMMA- and PS-SCNPs to that of the corresponding precursors, as a function of the X-linker amount in the precursors. The exponents have been obtained by fitting SEC results to a power-law $R_{H}=K_{H} M^{v}$ (see ref. 25 and Table 1). Data obtained from MD simulations concerning the dependence of $\left\langle R_{g}{ }^{2}\right\rangle^{1 / 2}$ on $M$ are also included in Figure 1 for comparison, by assuming that the dependences of $\left\langle R_{g}{ }^{2}\right\rangle^{1 / 2}$ and $R_{H}$ on $M$ are similar. $^{10,11}$ 
Table 1. Parameters of the $R_{H}$ and [ $\eta$ ] scaling power-laws, for PMMA- and PS-SCNPs as a function of the X-linker amount in the precursor. ${ }^{\text {a }}$

\begin{tabular}{|c|c|c|c|c|c|c|}
\hline \multirow[b]{2}{*}{ Entry } & \multirow[b]{2}{*}{$\begin{array}{c}\text { SCNP } \\
\text { type }\end{array}$} & \multirow[b]{2}{*}{$\begin{array}{l}\text { X-linker } \\
\text { amount } \\
(\text { mol\%) }\end{array}$} & \multicolumn{2}{|c|}{$\boldsymbol{R}_{H}=\boldsymbol{K}_{H} \boldsymbol{M}^{v}$} & \multicolumn{2}{|c|}{$[\eta]_{\mathrm{calc}}=\boldsymbol{K}_{\eta} \boldsymbol{M}^{a}$} \\
\hline & & & $K_{H}$ & $v$ & $K_{\eta}$ & $a$ \\
\hline 1 & \multirow[t]{4}{*}{ PMMA } & 10 & $1.67 \times 10^{-2}$ & 0.52 & $2.94 \times 10^{-2}$ & 0.56 \\
\hline 2 & & 15 & $1.93 \times 10^{-2}$ & 0.50 & $4.53 \times 10^{-2}$ & 0.51 \\
\hline 3 & & 20 & $1.94 \times 10^{-2}$ & 0.50 & $4.61 \times 10^{-2}$ & 0.50 \\
\hline 4 & & 25 & $2.01 \times 10^{-2}$ & 0.49 & $5.12 \times 10^{-2}$ & 0.47 \\
\hline 5 & \multirow[t]{4}{*}{ PS } & 10 & $1.92 \times 10^{-2}$ & 0.51 & $4.46 \times 10^{-2}$ & 0.53 \\
\hline 6 & & 15 & $2.04 \times 10^{-2}$ & 0.49 & $5.35 \times 10^{-2}$ & 0.47 \\
\hline 7 & & 20 & $2.12 \times 10^{-2}$ & 0.48 & $6.01 \times 10^{-2}$ & 0.44 \\
\hline 8 & & 25 & $2.18 \times 10^{-2}$ & 0.47 & $6.53 \times 10^{-2}$ & 0.41 \\
\hline
\end{tabular}

${ }^{\mathrm{a}} R_{H}$ and $M$ data obtained from SEC measurements (ref. 25).

A good agreement is observed between experimental and coarse-grained MD simulation data, supporting that the behavior illustrated in Figure 1 is a general behavior for SCNPs. The plateau at large X-linker fraction is related to local globulation events that take place during SCNP formation. As recently discussed, ${ }^{10,11,25}$ increasing the amount of $\mathrm{X}$-linkers beyond some level just increases the number of these events, which are inefficient for global folding, and hence do not further lower the scaling exponent of the SCNP.

As mentioned above, the analysis of the SEC data for the SCNPs provides the values of $K_{H}$ and $v$ in the scaling law $R_{H}=$ $K_{H} M^{v}$. On the other hand, the intrinsic viscosity is related to the viscosimetric radius $\left(R_{\eta}\right)$ through the Einstein viscosity law ${ }^{1,2}$ : $[\eta]=(10 \pi / 3) N_{A}\left(R_{\eta}^{3} / M\right)$. By combining the former expressions with the usual approximation $R_{\eta} \approx R_{H}$ we obtain the effective MHS equation $[\eta]=K_{\eta} M^{a}$, where $K_{\eta}=(10 \pi / 3) N_{A} K_{H}{ }^{3}$ and $a=$ $3 v-1$. Hence, by using the experimental values of $K_{H}$ and $v$ we can predict the molecular-weight dependence of the intrinsic viscosity of the SCNPs. The predicted values of $K_{\eta}$ and $a$ for PMMA- and PS-SCNPs, as a function of the amount of reactive $\mathrm{X}$-linker functional groups in the precursor, are summarized in Table 1. In what follows we will denote the values of the intrinsic viscosity calculated by this simple approach as $[\eta]_{\text {calc }}$, to distinguish them from the experimental data directly measured by viscosimetry, $[\eta]_{\text {exp }}$.

Figure 2 shows a comparison between theoretical, $[\eta]_{\text {calc }}$, and experimental data, ${ }^{26-28}[\eta]_{\text {exp }}$, of several SCNPs of different chemical nature. As can be seen, there is a reasonable agreement between $[\eta]_{\exp }$ and $[\eta]_{\text {calc }}$ data for $[\eta]_{\exp }>5 \mathrm{ml} / \mathrm{g}$, with an average standard deviation between both data sets of 12 $\%$, which may be attributed to the approximation of using $R_{H}$ data instead of $R_{\eta}$ data for the calculation of $[\eta]_{\text {calc }}$.

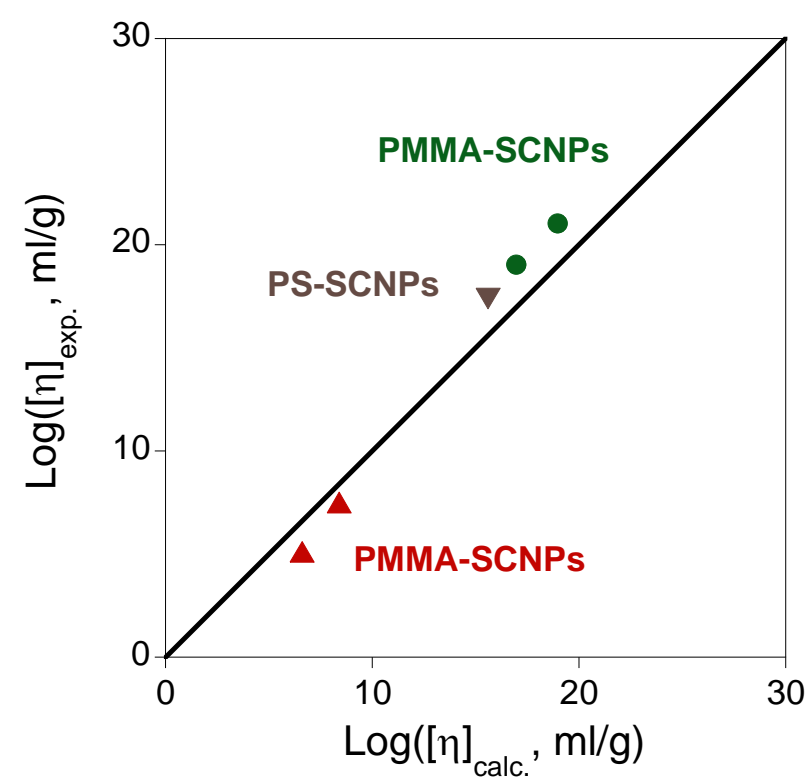

Fig. 2. Comparison of experimental intrinsic viscosity data, $[\eta]_{\exp }{ }^{26-28} v s$. theoretical values $[\eta]_{\text {calc }}$ for SCNPs of different chemical nature and molecular weight (see text and Table 1 ) The line corresponds to the case $[\eta]_{\text {calc }} \equiv[\eta]_{\text {exp }}$. Error bars for the experimental data are comparable to the size of the symbols.

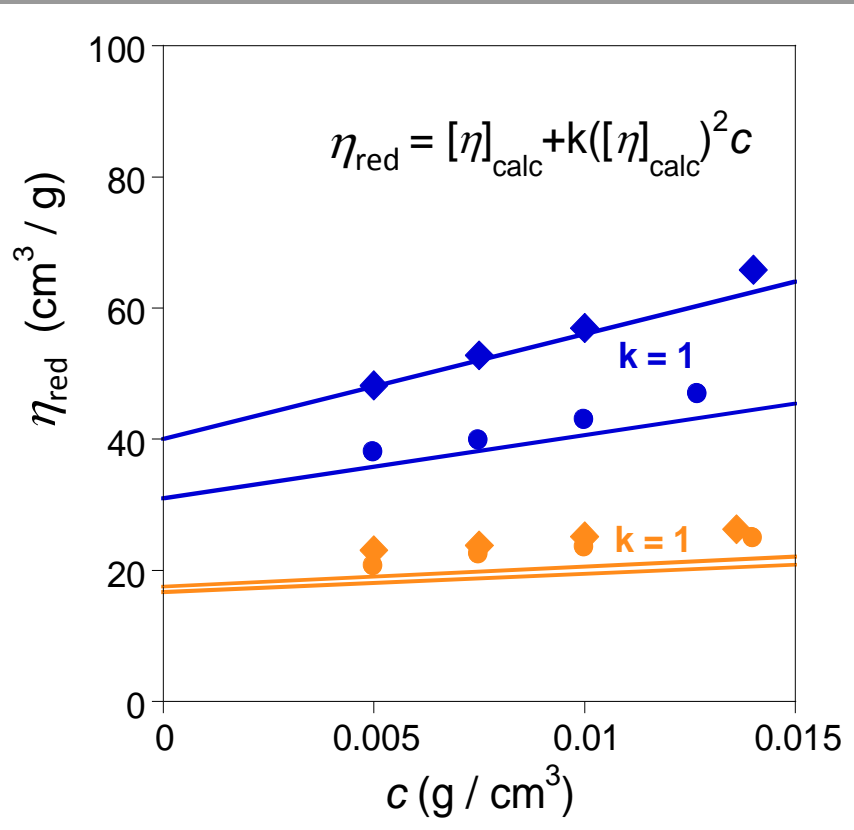

Fig. 3. Data for the reduced viscosity, $\eta_{\mathrm{red}}=\left(\eta-\eta_{\mathrm{s}}\right) / c \eta_{\mathrm{s}}$, as a function of the concentration $c$, for PMMA-SCNPs with the same X-linker fraction of $15 \%$ but different molecular weight (orange diamons: $150 \mathrm{kDa}$; orange circles: $100 \mathrm{kDa}$ ), and for their corresponding precursors (blue diamons: $150 \mathrm{kDa}$; blue circles: 100 $\mathrm{kDa}$ ). The experimental viscosimetry data were reported by Beck et al. in ref. 26, and have been sampled from Fig. 4 of that work. As usual, the experimental intrinsic viscosity is determined as the value of the reduced viscosity in the limit of zero concentration, $c=0$, by fitting the data to the Huggins equation ${ }^{1,2} \eta_{\text {red }}=$ $[\eta]_{\exp }+k\left([\eta]_{\exp }\right)^{2} c$. This leads to the intriguing result ${ }^{26}$ that the $[\eta]_{\exp }$ values for the SCNPs are very similar despite being very different for the corresponding precursors. This observation is nicely captured (solid lines) by the simple model employed in this work. The lines are obtained by using the theoretical values of $[\eta]_{\text {calc }}$ (see main text) in the Huggins equation, with a typical factor ${ }^{31} k=1$. For the SCNPs we use $K_{\eta}$ and $a$ from entry 2 of Table 1. 
The agreement between $[\eta]_{\exp }$ and $[\eta]_{\text {calc }}$ is expected to be improved significantly by using more elaborated theoretical approaches as, e.g., the recently developed partially permeable sphere model. ${ }^{29-30}$ Still, the use of a simple model treating SCNPs in solution as spheres of effective hydrodynamic radius $R_{H}$, and assuming $R_{H} \approx R_{\eta}$, provides a straightforward explanation to the observation by Beck et al. ${ }^{26}$ of very similar values of $[\eta]$ for PMMA-SCNPs that are synthesized from precursors having very different molecular weights and consequently very different values of $[\eta]$ (see Figure 3 ).

\section{Comparison of the intrinsic viscosity of SCNPs $v s$. star, hyperbranched and dendrimeric polymers}

The particular nanoscopic architecture of SCNPs accounts for the intriguing viscosity behaviour shown by these nanoobjects in dilute solution, which can be clearly appreciated in Figure 3. More interesting is, however, the comparison of $[\eta]$ values of SCNPs versus the $[\eta]$ values of star, hyperbranched and dendrimeric polymers of the same chemical nature and molecular weight. This comparison aids to unraveling the effect of the complex topology of SCNPs on the flow properties of their diluted solutions.

The different panels in Fig. 4 compare experimental results for the intrinsic viscosity of SCNPs (blue symbols) and the mentioned branched polymers (low-functionality stars, hyperbranched and dendrimers; orange symbols) with the same chemical structure. Error bars for the experimental data are comparable to the size of the symbols. The blue lines in the panels represent the theoretical intrinsic viscosities $[\eta]_{\text {calc }}$ provided by the employed model for the SCNPs, and computed from entries in Table 1 (see caption of Fig. 4 for details). We include theoretical results ranging from a "lower limit" of $\mathrm{X}$ linker fractions of interest $(\sim 10 \mathrm{~mol} \%)$ to large fractions approaching the plateau in the ratio $v_{\mathrm{SCNP}} / v_{\text {precursor }}$ (see Fig. 1). The green lines are the experimental power-laws $[\eta]_{\exp }$ obtained from viscosimetry measurements of the corresponding linear polymers.

Fig. 4a compares the $[\eta]$ vs. $M$ behavior of PMMASCNPs $^{26,28}$ and 6-arm PMMA stars. ${ }^{32}$ The star-shaped materials were synthesized by Chen et al. through group transfer polymerization using phosphazene base, showing very narrow molecular weight distribution $(1.06<Ð<1.16) .^{32}$ As can be seen in Fig. 4a, at any given value of $M$ the SCNPs display significantly lower values of $[\eta]$ when compared to 6-arm PMMA stars of the same mass (e.g., for $M \approx 100 \mathrm{kDa}$, $[\eta]_{\mathrm{exp}}(\mathrm{PMMA}-\mathrm{SCNPs}) \approx 18 \mathrm{ml} / \mathrm{g}^{26}$ vs. [ $\left.\eta\right]_{\exp }(6$-arm PMMA stars) $\approx 32 \mathrm{ml} / \mathrm{g}^{32}$ ). Upon increasing the amount of X-linkers in the precursor of the SCNP from $10 \mathrm{~mol} \%$ (solid blue line) to $15 \mathrm{~mol} \%$ (blue dashed line) and to $25 \mathrm{~mol} \%$ (blue dotted line), a progressive decrease in the value of $[\eta]$ is predicted, in good agreement with experimental results. A similar behaviour is expected for PS-SCNPs when compared to 6-arm PS stars, ${ }^{16}$ as illustrated in Fig. 4b. Once again, a significant reduction in $[\eta]$ is predicted upon increasing the amount of $\mathrm{X}$-linkers in the precursor from $10 \mathrm{~mol} \%$ (solid line in Fig. 4b) to $20 \mathrm{~mol} \%$ (dashed line) although, to the best of our knowledge, no experimental data are available to validate this trend.

Fig. 4c compares the $[\eta]$ vs. $M$ behavior of PMMA-SCNPs and fractionated hyperbranched (HB)-PMMA chains synthesized by Simon et al. through self-condensing group transfer copolymerization. ${ }^{33}$ We observe that at low molecular weight $(M<100 \mathrm{kDa})$ SCNPs have lower values of $[\eta]$ when compared to those of HB-PMMA chains of equivalent $M$ (e.g., for $M \approx 25 \mathrm{kDa},[\eta]_{\mathrm{exp}}(\mathrm{PMMA}-\mathrm{SCNPs}) \approx 8 \mathrm{ml} / \mathrm{g}^{28} v s$. $[\eta]_{\text {exp }}($ HB-PMMA chains $) \approx 12 \mathrm{ml} / \mathrm{g}^{33}$ ). Conversely, due to the lower value of the $a$ exponent in the MHS equation for HBPMMA chains when compared to SCNPs, the opposite behavior is expected at very high values of $M$. Once again, a similar behavior is predicted for PS-SCNPs although in this case the molecular weight at which the MHS equations of HBPS chains ${ }^{18}$ and SCNPs (10 mol\% X-linker in the precursor) cross each other is $>10^{6}$ Da (Fig. $4 d$ ).

The behavior of dendrimeric polymers in dilute solution is peculiar since, as mentioned above, a maximum in the $[\eta] v s . M$ plot is observed for these nano-objects (see Fig. 4e and 4f). ${ }^{34,35}$ As a consequence, even if SCNPs of relatively low molecular weight have values of $[\eta]$ similar or even lower than those of dendrimeric polymers of equivalent $M$, upon increasing the molecular weight this trend is clearly reversed, as illustrated in Fig. $4 \mathrm{e}$ and $4 \mathrm{f}$. In particular, the specific value of $M$ at which the $[\eta]$-curve of the dendrimeric polymer crosses the line of the SCNP strongly depends (varying even an order of magnitude) on the amount of X-linkers in the precursor.

\section{Conclusions}

SCNPs in solution adopt open, sparse morphologies resembling those of intrinsically disordered proteins with locally compact portions connected by flexible segments, as revealed by recent SANS, SAXS and MD simulations. In this work, a simple power-law relation between the intrinsic viscosity $[\eta]$ and the molecular weight has been derived for SCNPs, as a function of their fraction of $\mathrm{X}$-linkers, by combining the Einstein equation for the intrinsic viscosity and experimental SEC data for the hydrodynamic radii. It is worth of mention that the own SCNP topology leads to a particular $M_{w}$-dependence of $\mathrm{R}_{\mathrm{H}}$. The underlying physics involved, as in any other polymer solution, is Zimm-like dynamics with a mapping of the $R_{H}$-dependence of $[\eta]$ through the Einstein model. The good agreement between theoretical and experimental values of $[\eta]$ validates this approach.

The results reported here provide a global picture for the intrinsic viscosity of SCNPs in solution. As a consequence of their complex nanoscopic architecture, the intrinsic viscosities of SCNPs are systematically smaller than those of linear chains and low-functionality stars. However, when compared with hyperbranched and dendrimeric polymers, a complex behaviour is found, this being highly dependent on the molecular weight and amount of X-linker sites in the SCNP. 

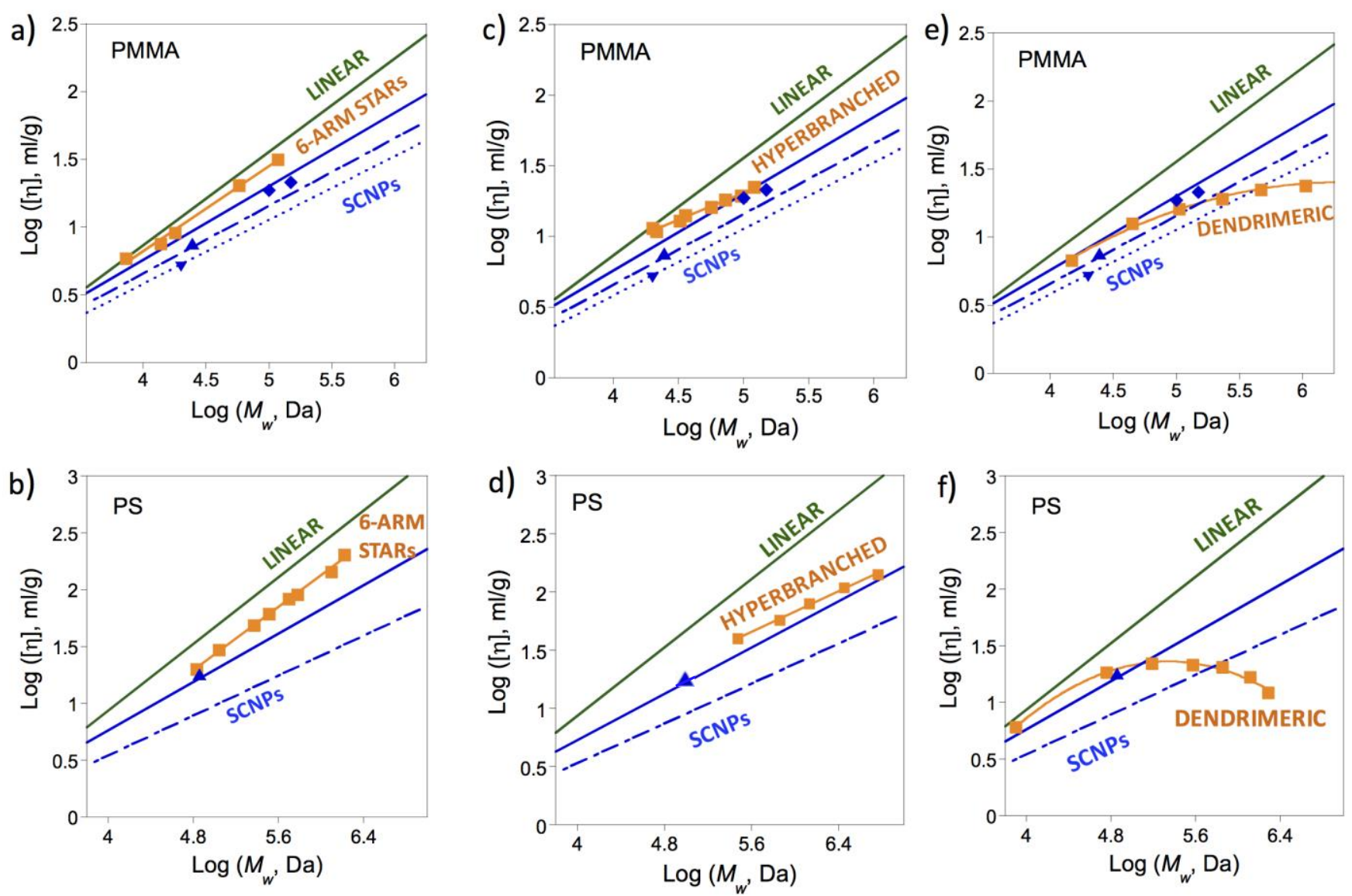

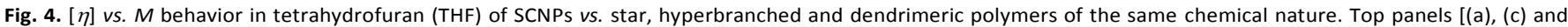

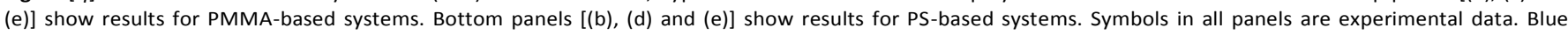

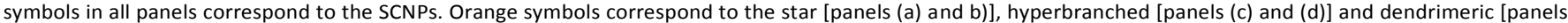

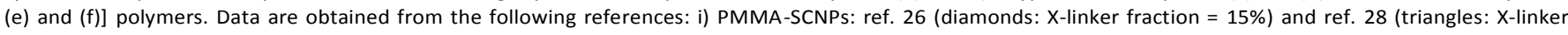

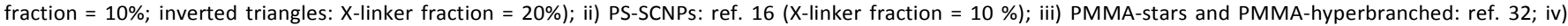

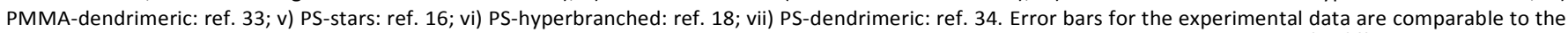

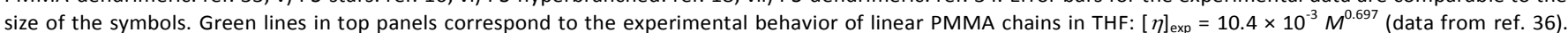

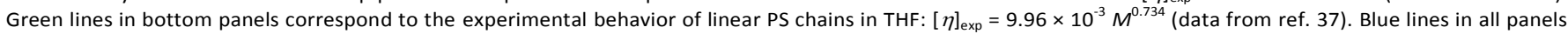

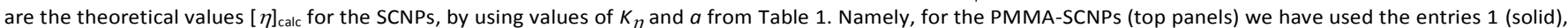

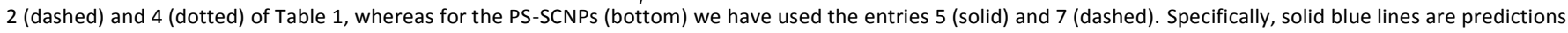

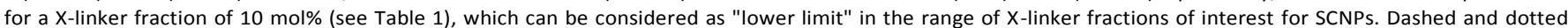

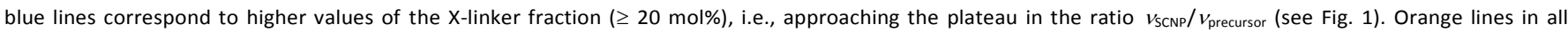

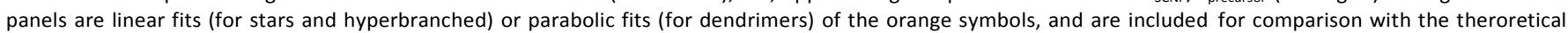
curves of the SCNPs (blue lines).

\section{Acknowledgements}

Financial support from the Projects MAT2012-31088 (MINECO), T-654-13 (GV) and S-PE13UN034 (GV) is acknowledged. I. P.-B. acknowledges CSIC for her JAEPREDOC grant. We acknowledge CSUC (Spain) for generous allocation of CPU time.

\section{Notes and references}

${ }^{a}$ Materials Physics Center MPC, Paseo Manuel de Lardizabal 5, E-20018 San Sebastián, Spain.

${ }^{b}$ Centro de Física de Materiales (CSIC, UPV/EHU), Paseo Manuel de Lardizabal 5, E-20018 San Sebastián, Spain.
${ }^{c}$ Departamento de Física de Materiales, Universidad del País Vasco (UPV/EHU), Apartado 1072, E-20080 San Sebastián, Spain.

${ }^{d}$ Donostia International Physics Center, Paseo Manuel de Lardizabal 4, 20018 San Sebastián, Spain.

${ }^{e}$ IKERBASQUE Basque Foundation for Science, Alameda de Urquijo 36, E-48011 Bilbao, Spain.

1 M. Doi and S. F. Edwards, The Theory of Polymer Dynamics, Oxford University Press, Oxford, UK, 1986

2 M. Rubinstein and R. H. Colby, Polymer Physics, Oxford University Press, Oxford, UK, 2003. 
3 O. Altintas and C. Barner-Kowollik, Macromol. Rapid Commun., 2012, 33, 958-971.

4 J. A. Pomposo, Polym. Int., 2014, 63, 589-592.

5 A. Sanchez-Sanchez, I. Perez-Baena and J. A. Pomposo, Molecules, 2013, 18, 3339-3355.

6 A. Sanchez-Sanchez and J. A. Pomposo, Part. Part. Syst. Charact., 2014, 31, 11-23.

7 A. Sanchez-Sanchez, S. Akbari, A. Etxeberria, A. Arbe, U. Gasser, A. J. Moreno, J. Colmenero and J. A. Pomposo, ACS Macro Lett., 2013, 2, 491-495.

8 I. Perez-Baena, F. Barroso-Bujans, U. Gasser, A. Arbe, A. J. Moreno, J. Colmenero and J. A. Pomposo, ACS Macro Lett., 2013, 2, 775-779.

9 A. Sanchez-Sanchez, S. Akbari, A. J. Moreno, F. Lo Verso, A. Arbe, J. Colmenero and J. A. Pomposo, Macromol. Rapid Commun., 2013, 34, 1681-1686.

10 A. J. Moreno, F. Lo Verso, A. Sanchez-Sanchez, A. Arbe, J. Colmenero and J. A. Pomposo, Macromolecules, 2013, 46, 97489759.

11 F. Lo Verso, J. A. Pomposo, J. Colmenero and A. J. Moreno, Soft Matter, 2014, 10, 4813-4821.

12 B. H. Zimm and W. H. Stockmayer, J. Chem. Phys., 1949, 17, 13011314.

13 B. H. Zimm and R. W. Kilb, J. Polym. Sci., 1959, 37, 19-42.

14 W. H. Stockmayer and M. Fixman, Ann. N.Y. Acad. Sci., 1953, 57, 334-352.

15 N. Khasat, R. W. Pennisi, N. Hadjichristidis and L. J. Fetters, Macromolecules, 1988, 21, 1100-1106.

16 L. A. Utracki and J. E. Roovers, Macromolecules, 1973, 6, 366-372.

17 J. Roovers and S. Bywater, Macromolecules, 1974, 7, 443-449.

18 L. Li, Y. Lu, L. An and C. Wu, J. Chem. Phys., 2013, 138, 114908.

19 J. M. J. Fréchet and D. A. Tomalia, Dendrimers and Other Dendritic Polymers, Ch. 14, Wiley, UK, 2001.

20 T. H. Mourey, S. R. Turner, M. Rubinstein, J. M. J. Fréchet, C. J. Hawker and K. L. Wooley, Macromolecules, 1992, 25, 2401-2406.

21 A. M. Naylor, W. A. Goddard III, G. Keifer and D. A. Tomalia, J. Am. Chem. Soc., 1989, 111, 2339-2341.

22 A. Einstein, Ann. Phys., 1911, 339, 591-592.

23 J. M. J. Fréchet, Science, 1994, 263, 1710-1715.

24 M. Jeong, M. E. Mackay, R. Vestberg and C. J. Hawker, Macromolecules, 2001, 34, 4927-4936.

25 J. A. Pomposo, I. Perez-Baena, F. Lo Verso, A. J. Moreno, A. Arbe and J. Colmenero, ACS Macro Lett., 2014, 3, 767-772.

26 J. B. Beck, K. L. Killops, T. Kang, K. Sivanandan, A. Bayles, M. E. Mackay, K. L. Wooley and C. J. Hawker, Macromolecules, 2009, 42, 5629-5635.

27 B. T. Tuten, D. Chao, C. K. Lyon and E. B. Berda, Polym. Chem., 2012, 3, 3068-3071.

28 P. G. Frank, B. T. Tuten, A. Prasher, D. Chao and E. B. Berda, Macromol. Rapid Commun., 2014, 35, 249-253.

29 Y. Y. Yu, T. F. Shi, L. J. An, Z.-G. Wang, EPL, 2012, 97, 64003(16).

30 Y. Lu, L. An, Z.-G. Wang, Macromolecules, 2013, 46, 5731-5740.

31 R. Pamies, J. G. Hernández-Cifre, M. C. López-Martínez, J. G. García de la Torre, Colloid. Polym. Sci., 2008, 286, 1223-1231.

32 Y. Chen, K. Fuchise, A. Narumi, S. Kawaguchi, T. Satoh and T. Kakuchi, Macromolecules, 2011, 44, 9091-9098.
33 P. F. W. Simon, A. H. E. Müller and T. Pakula, Macromolecules, 2001, 34, 1677-1684.

34 A. Hirao and H.-S. Yoo, Polym. J., 2011, 43, 2-17.

35 R. Matmour and Y. Gnanou, J. Am. Chem. Soc., 2008, 130, 13501361.

36 L. J. Fetters, N. Hadjichristidis, J. S. Lindner and J. W. Mays, J. Phys. Chem. Ref. Data, 1994, 23, 619-640.

37 H. L. Wagner, J. Phys. Chem. Ref. Data, 1987, 16, 165-173. 